\title{
The unfinished history of aspirin hypersensitivity - in memory of Professor Andrew Szczeklik
}

\author{
Marek Sanak \\ 2nd Department of Medicine, Jagiellonian University Medical College, Kraków, Poland
}

Correspondence to: Prof. Marek Sanak, MD, PhD, II Katedra Chorób Wewnętrznych, ul. Skawińska 8, 31-066 Kraków, e-mail: nfsanak@cyf-kr.edu.pl Received: March 23, 2012. Accepted: March 23, 2012 Pol Arch Med Wewn. 2012; 122 (Special Issue): $42-45$

Copyright by Medycyna Praktyczna, Kraków 2012
Salicylic acid has been used since thousands of years as antiphlogistic, antipyretic, or pain relief remedy. The formula was mainly concocts of willow bark or myrtle (wintergreen) oil. A German chemist, Felix Hoffman, when looking for a better substitute of sodium salicylate for his father who suffered arthritis, rediscovered acetylsalicylic acid in 1897. He was fortunate that Bayer company, which he worked for, decided to marked this product around 1899. The origin of the commercial name, Aspirin, was interesting. Spiric acid is an alternate name to salicylic acid because of its presence in spirea shrubs. "A" came from the acetyl group. The interest of the company in a new painkiller was due to the fact that the previous product which hit the market turned out highly addictive. Its name was Heroin. Adverse reactions, precipitated following ingestion of aspirin, were described as early as in 1902. A French physician, Fernand Vidal, described a triad of asthma, nasal polyposis and aspirin hypersensitivity in 1922. The natural course of the syndrome was further refined by Samters and Beers in 1968. This syndrome is currently recognized as a particular phenotype of asthma. It affects up to $15 \%$ intrinsic asthma subjects. There is also a cutaneous syndrome, aspirin-triggered urticarial/angioedema, which is much less predictable in its course.

In 1975, Andrew Szczeklik, Richard Gryglewski, and Grażyna Czerniawska-Mysik published in the British Medical Journal ${ }^{1}$ their report on oral provocations of 11 asthmatic subjects manifesting hypersensitivity to aspirin. They demonstrated a cross-reactivity between aspirin and other non-steroidal anti-inflammatory drugs (NSAIDs), namely indomethacin, mefenamic, and flufenamic acids and phenylbutazone. Interestingly, salicylamide, paracetamol and two other now discontinued antipyretics turned out well tolerated. For the first time, a consistent relation has been demonstrated between in-vitro parameters of NSAIDs potency and the provoking dose which decreased the lung peek expiratory flow. One of these laboratory parameters was inhibitory concentration $50 \%$ which inhibited the production of prostaglandin $\mathrm{E}_{2}\left(\mathrm{PGE}_{2}\right)$ in a bioassay using microsomal fraction of bovine seminal vesicles for $\mathrm{PGE}_{2}$ biosynthesis and the rat stomach strip as a sensor. The comparison of the drugs was controlled by a second parameter. In a drug-binding assay, a small fluorescent tagging molecule was displaced from the albumin by a drug. Providing simplicity of these laboratory assays, results were impressively coherent. There was a significant positive correlation between the highest tolerated dose of the drug and its concentration inhibiting $\mathrm{PGE}_{2}$ biosynthesis, even when compensated for binding of the drug to the albumin. This report was published only a few years after Sir John Vane's observation that aspirin-like drugs inhibit prostaglandin synthesis, ${ }^{2}$ and one year before the first purified preparations of arachidonic acid cyclooxygenase enzyme were obtained. Thus, the cyclooxygenase theory of aspirin-induced asthma was born contemporary with the major discoveries in the field of arachidonic acid metabolism. In the following 35 years Andrew Szczeklik, though highly active in many others fields of medical research, remained fascinated with the phenomenon of asthma hypersensitive to aspirin and other NSAIDs. When I joined his team in 1995, he initiated a novel molecular biology laboratory in a modern facility building, an extension to the old community hospital on the Skawinska Street at the Jewish Quarter of Kazimierz. There, in 1972, Andrew Szczeklik became the Head of the Clinic of Allergology, later renamed to the Department of Medicine. This was the time of a fast advancement in human genetics. In 1996, stimulated by a publication on cloning of the leukotriene $C 4$ synthase gene $^{3}$ we screened DNA from patients with aspirin hypersensitivity and described a common genetic variant, which seemed associated with the disease. ${ }^{4}$ Andrew Szczeklik was proud of this discovery because of the award he received during The Lancet's conference on The Challenge of Asthma in Tours, France, 1997. This was one of 


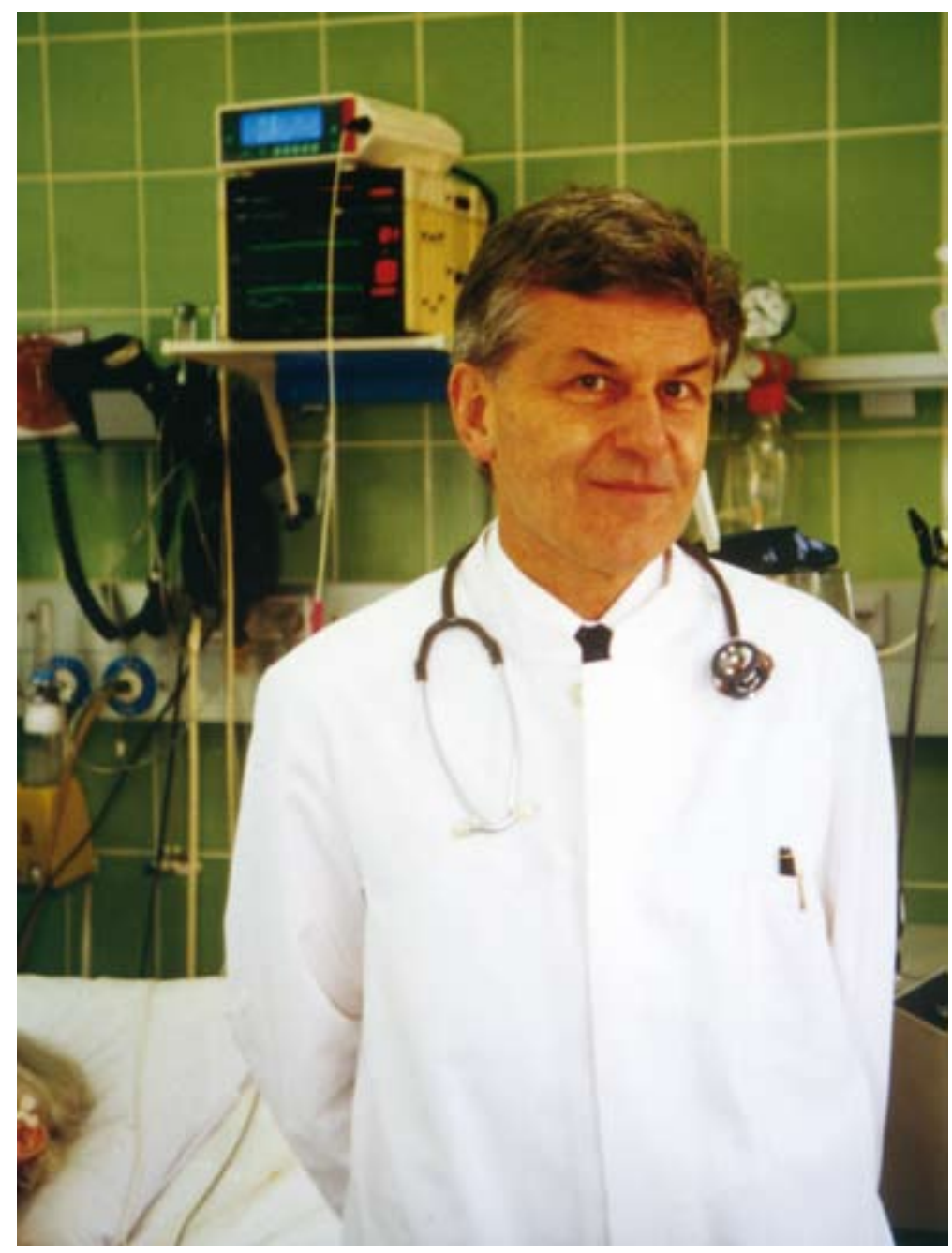

FIGURE 32 Professor Szczeklik at the intensive care unit, 2nd Department of Internal Medicine, Jagiellonian University Medical College, Krakow, 1990s

the first non-coding polymorphisms located in the promoter region of the gene, which functional associations have been replicated in the following years. The polymorphism has an impact both on biosynthetic capacity of cysteinyl leukotrienes and clinical response to leukotriene-modifying drugs. From the perspective of time, the magnitude of the association was probably overestimated by the selection of the most severe asthmatic subjects; however, it remained detectable in several studies on cysteinyl leukotrienes dependent phenotypes. Cysteinyl leukotrienes, another class of arachidonic acid metabolites (eicosanoids) of 5-lipoxygenation pathway, were incriminated for mediating bronchoconstriction since their very first discovery in the model of guinea pig lung. Andrew Szczeklik's team confirmed that urinary excretion of the end metabolite leukotriene $\mathrm{E}_{4}$ is a useful and robust marker of aspirin hypersensitivity. ${ }^{5}$ But it was also noticed that an unexplained interaction existed between eicosanoids originating from a separate metabolic pathways, i.e., 5-lipoxygenase and cyclooxygenase of arachidonic acid. Inhibition of cyclooxygenase activity resulted in overproduction of cysteinyl leukotrienes. Clinical experiments, in which a water soluble aspirin was instilled into segmental bronchi, demonstrated also a simultaneous decrease

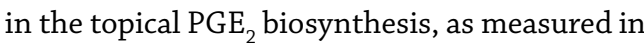
bronchoalveolar lavage fluid. ${ }^{6}$ The first attempt to explain this reciprocal changes of the two eicosanoid classes was really a mechanistic one. Perhaps, the intracellular substrate due to cyclooxygenase inhibition was shifted into an alternate pathway of arachidonic acid 5-lipoxygenase, and further downstream into cysteinyl leukotrienes biosynthesis. This possibility has not been falsified definitely yet. However, clinical evidences suggested that highly selective cyclooxygenase-2 inhibitors, coxibs, were well tolerated by aspirin-hypersensitive asthmatics, despite a significant decrease in systemic biosynthesis of $\mathrm{PGE}_{2}$ following the administration of these drugs. When systemic biosynthesis of $\mathrm{PGE}_{2}$ was monitored by measurements of urinary metabolites of this prostaglandin: 13, 14-hydroxy, 15-keto-PGE ${ }_{2}$ and tetranor-PGEM, another unexplained discrepancy was observed. Administration of celecoxib suppressed urinary metabolites of $\mathrm{PGE}_{2}$ and did not precipitate bronchoconstriction. But administration of aspirin was a trigger for dyspnea; however, these asthmatics continued to have their urinary $\mathrm{PGE}_{2}$ metabolites elevated. ${ }^{7}$ Since average oral dose of aspirin precipitating asthmatic attack was less than $200 \mathrm{mg}$, lack of depression in systemic $\mathrm{PGE}_{2}$ biosynthesis could be explained by a rather weak inhibitory effect of aspirin on both cyclooxygenase isoenzymes. However, overproduction of $\mathrm{PGE}_{2}$ noticed using measurements of the urinary excretion of these metabolites was puzzling on the whole organism level. At the same time of oral aspirin challenge, an increase of cysteinyl leukotriene $\mathrm{E}_{4}$ in urine was the expected biomarker of hypersensitivity reaction. These results were definitely against the intracellular arachidonic shift, but also questioned the validity of cyclooxygenase theory of aspirin hypersensitivity.

During the last decade, there was a tremendous progress in laboratory methods used for identification and measurement of eicosanoids. Because of their lipophilic properties and a chemical structure similar to each other, a chromatography-mass spectrometry turned out to be the only method adequate for the research purposes. Andrew Szczeklik equipped his laboratory with a gas chromatography-mass spectrometry instrument in 1996. Measurements using this apparatus required a skillful preparation of samples because analyzed molecules had to be modified chemically - derivatized by esterification or etherification - to acquire volatile properties. The laboratory staff spent several weeks in Nashville and New York to learn the tricks and tips on measurements of eicosanoids in biological samples. This was possible only due to personal contacts and friendship of Andrew Szczeklik with the best experts in physiopathology of eicosanoids: James Sheller, Colin Funk, and John McGiff.

To illustrate the progress in analytical methods of eicosanoids one can compare bioassay used by 
Richard Gryglewski in 1975, the capacity of which to produce $\mathrm{PGE}_{2}$ in vitro was around $1.5 \mathrm{mg} / \mathrm{ml}$, with the lowest detection level of the measurement achieved with GC-MS, which equals $0.3 \mathrm{pg}$ / $\mathrm{ml}$. This was a 5 billion-fold increase in the sensitivity of the method. Similar advancement was obtained using another analytical platform acquired by Andrew Szczeklik in 2007. This was a modern high-perfomance liquid chromatography - tandem mass spectrometer. The instrument enabled us to measure precisely not only cysteinyl leukotrienes, but also about 40 other eicosanoid metabolites in a variety of biological fluids. Thanks to the European Community support and once again personal contacts with a prominent Norwegian specialist in asthma and pulmonology Kai-Haakon Carlsen, all these analytical methods were rigorously exercised and validated. This also created some new opportunities to study local biosynthesis of eicosanoids within the lung. During regular breathing a small fraction of epithelial lining fluid of the lung is admixed into the air and forms an aerosol. These particles of fluid have diameter below $1 \mu \mathrm{m}$, but can be condensed together with a water vapor using a simple cooling device. The obtained fluid is named an exhaled breath condensate. Within $15-20$ minutes of breathing as much as $3-4 \mathrm{ml}$ of the condensate is collected, which contains admixture of nonvolatile molecules present on the lung epithelial surface. The main obstacle for assessment of the exhaled breath condensate content is a dilution with water, which is unpredictable. We managed to compensate for a variance of the exhaled breath condensate dilution by a simultaneous measurement of a free and abundant saturated fatty. Several tests showed that palmitic acid has a constant concentration in epithelial lining fluid, and the acid can be used to correct for the dilution factor. ${ }^{8}$ Using this method, we demonstrated that alterations in metabolic profile of eicosanoids created a distinct handprint of aspirin exacerbated respiratory disorder. Not only 5-lipoxygenase pathway seemed upregulated but also 15-lipoxygenase metabolites were elevated. Moreover, overproduction of $\mathrm{PGD}_{2}$ and $\mathrm{PGE}_{2}$ metabolites in the lung of aspirin hypersensitive asthmatics was present without any aspirin provocation and despite their stable clinical condition. Some new classes of eicosanoids, such as lipoxins, seemed also downregulated in these patients; however, our analytical platform has been still imperfect to measure them precisely. ${ }^{9}$ The next task was to monitor changes in the profile of eicosanoids from the exhaled breath condensate during aspirin provocation. These results were collected within the last year. Although we discussed them with Andrew Szczeklik, the manuscript was published just a few days before his death. To our disappointment, no significant changes in eicosanoid levels were noted following aspirin precipitated bronchoconstriction, when compared to the pre-challenge levels. ${ }^{10}$ Some previous observations, such as elevated concentration of $\mathrm{PGE}_{2}$ metabolites, were confirmed in the exhaled breath condensate. It seemed that either cysteinyl leukotrienes were released into bloodstream rather than to the epithelial lining fluid, or their rise on the systemic level resulted from biosynthesis within subepithelial layers of bronchi. We have noticed also some interesting regularities in appearance of additional eicosanoid molecules, which we try to characterize now. Andrew Szczeklik had foreseen the need for a continuous pursuit of novel eicosanoid mediators. He submitted a grant application for a better class of mass-spectrometry platform. This application had a great chance of success because the call was addressed to the most prominent researchers. Within the last decade he was the top one in the field of aspirin hypersensitivity and the leader in the Polish national ranking of biomedical research scientists.

It is uncertain which direction the future research on aspirin hypersensitivity will take. We are continuing studies on novel eicosanoids possibly involved in the mechanism of aspirin-triggered bronchoconstriction. A generous gift of Andrew Szczeklik to us was his latest project. This is a joint research plan that involves the leading Swiss institute in research of asthma and allergic diseases - SIAF in Davos. Aspirin hypersensitivity has many features of persistent inflammatory disorder of the respiratory tract. The syndrome is acquired and progresses from upper respiratory symptoms to asthma accompanied by a chronic rhinosinusitis with nasal polyposis. The hypothesis of a persistent viral infection leading to aspirin-induced asthma was put forward by Andrew Szczeklik almost 25 years ago. ${ }^{11}$ During our study on rhinovirus infections in asthma, we described persistent rhinoviral RNA signatures in aspirinhypersensitive asthmatics. ${ }^{12}$ Within the same frame of Swiss-Polish collaboration, again supported by the European Community, we will employ much more sensitive and robust methods of detection of respiratory viruses developed by Krzysztof Pyrć from the Jagiellonian University. ${ }^{13}$ Cezmi Akdis who is a director of SIAF and our collaborator in this project offered his expertise in studies on innate immunity and immunoregulation in aspirin hypersensitivity. We will try to establish a model of the disease enabling replication of clinical findings on the cellular and molecular level. Some previous experiments showed that primary cell lines of respiratory epithelia, fibroblasts, or peripheral blood cells continue to express phenotypic features of aspirin hypersensitivity ex vivo. Thus, the story on aspirin hypersensitivity seems to continue as designed by Andrew Szczeklik. What we were not prepared for, has been to find his pristine courage in struggling with the problem that evaded elucidation for almost a century. 


\section{REFERENCES}

1 Szczeklik A, Gryglewski RJ, Czerniawska-Mysik G. Relationship of inhibition of prostaglandin biosyntheis by analgesics to asthma attacks in aspirin-sensitive patients. Br Med J. 1975; 1: 67-69.

2 Vane JR. Inhibition of prostaglandin synthesis as a mechanism of action for aspirin-like drugs. Nat New Biol. 1971; 231: 232-235.

3 Lam BK, Penrose JF, Freeman GJ, Austen KF. Expression cloning of a cDNA for human leukotriene $\mathrm{C} 4$ synthase, an integral membrane protein conjugating reduced glutathione to leukotriene A4. Proc Natl Acad Sci U S A 1994; 91: 7663-7667.

4 Sanak M, Simon HU, Szczeklik A. Leukotriene C4 synthase promotr polymorphism and risk of aspirin-induced asthma. Lancet. 1997; 350 : 1599-1600

5 Sanak M, Bochenek G, Faber J, et al. Elevated urinary leukotriene E4 excretion in asthma: a comparison of HPLC-mass spectrometry with ELISA Allergy. 2010; 663-664.

6 Szczeklik A, Stadek K, Dworski R, et al. Bronchial aspirin challenge causes specific eicosanoid response in aspirin-sensitive asthmatics. Am J Resp Crit Care Med. 1996; 154: 1608-1614.

7 Mastalerz L, Sanak M, Gawlewicz-Mroczka A, et al. Prostaglandin E2 systemic prodyction in patients with asthma with and without aspirin hypersensitivity. Thorax. 2008; 63: 27-34.

8 Sanak M, Gielicz A, Nagraba K, et al. Targeted eicosanoids lipidomic of exhaled breath condensate in healthy subjects. J Chromatogr B Analyt Technol Biomed Life Sci. 2010; 878: 1796-800.

9 Sanak M, Gielicz A, Bochenek G, et al. Targeted eicosanoid lipidomics of exhaled breath condensate provide a distinct pattern in the aspirin-intolerant asthma phenotype. J Allergy Clin Immunol. 2011; 127: 1141-1147.

10 Mastalerz L, Sanak M, Kumik J, et al. Exhaled eicosanoids following bronchial aspirin challenge in asthma patients with and without aspirin hypersensitivity. The pilot study. J Allergy. 2012; 2012: 696 792. Epub 2012.

11 Szczeklik A. Aspirin-induced asthma as a viral disease. Clin Allergy. 1988; $18: 15-20$

12 Woś M, Sanak M, Soja J, et al. The presence of rhinovirus in lower airways of patients withc bronchial asthma. Am J Resp Crit Care Med. 2008; 177: 1082-1089

13 Pyrć K, Sożek K, Wójcik K, et al. Use of sensitive, broad spectrum molecular assays and human airway epithelium cultures for detection of respiratory pathohens. PLoS One. 2012; 7: e32582. 